\title{
The Lung Immune Prognostic Index (LIPI) stratifies prognostic groups in advanced non-small cell lung cancer (NSCLC) patients
}

\author{
Mihaela Aldea ${ }^{1}$, Jose Carlos Benitez ${ }^{1}$, Laura Mezquita ${ }^{1,2,3}$ \\ ${ }^{1}$ Medical Oncology Department, Gustave Roussy, Villejuif, France; ${ }^{2}$ Translational Genomics and Targeted Therapeutics in Solid Tumors, August Pi i \\ Sunyer Biomedical Research Institute (IDIBAPS), Barcelona, Spain; ${ }^{3}$ Medical Oncology Department, Hospital Clínic, Barcelona, Spain \\ Correspondence to: Laura Mezquita, MD, PhD. Translational Genomics and Targeted Therapeutics in Solid Tumors Group, IDIBAPS, Barcelona, Spain; \\ Medical Oncology Department, Hospital Clínic, Calle Villarroel 170, Escalera 2, Planta 5, 08036, Barcelona, Spain. Email: 1mezquita@clinic.cat. \\ Provenance and Peer Review: This article was commissioned by the editorial office, Translational Lung Cancer Research. The article did not undergo external \\ peer review. \\ Comment on: Ruiz-Bañobre J, Areses-Manrique MC, Mosquera-Martínez J, et al. Evaluation of the lung immune prognostic index in advanced non-small \\ cell lung cancer patients under nivolumab monotherapy. Transl Lung Cancer Res 2019;8:1078-85.
}

Submitted Mar 25, 2020. Accepted for publication Apr 07, 2020.

doi: $10.21037 /$ tlcr.2020.04.14

View this article at: http://dx.doi.org/10.21037/tlcr.2020.04.14

In the therapeutic landscape of non-small cell lung cancer (NSCLC), immune-checkpoint inhibitors (ICI) have greatly improved the overall survival (OS) in the metastatic setting of non-oncogene addicted tumors, as well as in stage III disease, when given as consolidation therapy after chemoradiation (1). Unpreceded long responses have been seen with ICI, which raised for the first time a hope for cure even in a metastatic disease (2). However, not all patients respond and aggressive response patterns such as fastprogression or hyperprogression have been observed under ICI $(3,4)$. The identification of factors predicting for ICI response or resistance is thus of crucial importance as this might greatly impact patient survival.

Markers used in immuno-oncology, such as programmed death-ligand 1 (PD-L1) or tumor mutation burden (TMB), focus on the tumor while other tools, such as tumor infiltrating lymphocytes, interferon- $\gamma$ signatures, PD-L1 expression in immune cells and variations of circulating immune cell counts reflect the systemic inflammatory state and the host's immunologic state (5-7). In the last years, blood cell counts including immune cells have been actively investigated as part of different scores that are very easy to perform, such as the platelet-to-lymphocyte ratio (PLR), the neutrophil-to-lymphocyte ratio (neutrophils/lymphocytes) (NLR) or the derived neutrophil-to-lymphocyte ratio (dNLR) [neutrophils/(leukocytes - neutrophils)] (8).

The Lung Immune Prognostic Index (LIPI) was more recently developed by the association between the dNLR and the blood level of lactate dehydrogenase (LDH). LIPI has been initially developed in an attempt to predict response to immunotherapy and to guide treatment selection. In a retrospective analysis of 466 patients with advanced NSCLC treated with ICI and 162 treated exclusively with chemotherapy, LIPI was divided in three subsets of scores: good, intermediate and poor LIPI, based on the following cutoffs: $\mathrm{dNLR} \leq 3$ and $\mathrm{LDH} \leq$ upper limit of normal (ULN), dNLR $>3$ or LDH $>$ ULN, and dNLR $>3$ and LDH $>$ ULN (9). In the ICI cohort, the disease control rate (DCR), progression-free survival (PFS) and OS were significantly different for patients with poor, intermediate and good LIPI, respectively, with the poorest outcomes for the poor LIPI subgroup. Thus, median OS was 4.8 months (mo) (95\% CI: 3.6-7.7), 10.0 mo (95\% CI: 7.3-12.6) and $16.5 \mathrm{mo}$ (95\% CI: 11.4-34.0) for the poor, intermediate, and good LIPI groups, while median PFS was 2.0 mo (95\% CI: $1.7-4.0$ ), 3.7 mo (95\% CI: 3.0-4.8) and 6.3 mo (95\% CI: 5.0-8.0), respectively. Importantly, intermediate and poor LIPI were independently associated with disease progression at time of the first radiological examination, with an odds ratio of 2.20 (95\% CI: $1.26-$ 3.84; $\mathrm{P}=0.005$ ) and 3.04 (95\% CI: 1.46-6.36; $\mathrm{P}=0.003$ ), respectively. For the chemotherapy cohort, no differences were observed in OS and PFS between the three LIPI subgroups (9). The strong correlation between clinical 
outcomes and LIPI subsets indicated the prognostic role of LIPI in pretreated advanced NSCLC receiving ICI therapies, but not in the chemotherapy cohort. This has raised the question of a potential predictive value of LIPI for those patients treated with ICI.

Following this first report, the predictive role of LIPI has been investigated in other studies, but it has not been confirmed $(10,11)$. The prognostic impact however was consistent across all studies in patients treated with ICI or chemotherapy (10-13). In a pooled analysis with individual participant data of the BIRCH, FIR, OAK and POPLAR clinical trials $(\mathrm{N}=2,220)$, the three LIPI subsets were significantly associated with OS, PFS and response rate in patients treated with atezolizumab, as well as in patients that had docetaxel (10). Interestingly, in this study, atezolizumab showed better OS compared with docetaxel in the good [18.4 vs. $13.6 \mathrm{mo}$, respectively; hazard ratio (HR) 0.76, 95\% CI: 0.62-0.94] and intermediate LIPI subsets [11.3 vs. 8.9 mo, respectively; HR 0.75 , 95\% CI: 0.62-0.91], but not in the poor LIPI group [4.5 vs. 4.8 mo, respectively; HR 0.91, 95\% CI: 0.63-1.30]. Moreover, there was a lack of benefit with atezolizumab compared with docetaxel in nearly $11 \%$ of patients in the poor LIPI group, according to a biomarker interaction analysis by LIPI groups (14).

In another large pooled analysis of NSCLC patients enrolled in clinical trials who received ICI $(\mathrm{N}=1,368)$, chemotherapy $(\mathrm{N}=1,072)$ or targeted treatment $(\mathrm{N}=437)$, LIPI confirmed a prognostic value consistent with previous studies (11). A good LIPI score was associated with longer OS compared with a poor LIPI score for both the ICI (15.6 vs. 4.5 mo, HR 0.34; 95\% CI: 0.28-0.42) and for the chemotherapy cohort (10.4 vs. 5.3 mo, HR 0.49; $95 \%$ CI: 0.40-0.60). Similar to the study of Sorich et al., these data suggest that poor LIPI patients might not have improved outcomes with ICI (median OS $4.5 \mathrm{mo}$ ) as compared with chemotherapy (median OS $5.3 \mathrm{mo}$ ), but this hypothesis needs further evaluation in prospective studies. Also, this study was the first to assess the association between LIPI and clinical outcomes in NSCLC patients with EGFR- and $A L K$-driven NSCLC treated with tyrosine kinase inhibitors. In this setting, the median OS of patients was 46.5 mo (95\% CI: 37.7-not reached), 32.8 mo (95\% CI: 24.3-not reached) and $16.6 \mathrm{mo}$ (95\% CI: 12.4-20.0) in the good, intermediated and poor LIPI subgroups (11).

Additional studies explored the role of the LIPI in patients treated with ICI. In the study accompanying this editorial, Ruiz-Bañobre et al. evaluated LIPI in 153 advanced NSCLC patients from the Spanish Expanded Access, who received nivolumab monotherapy after prior treatment (12). Similar to the pivotal study of LIPI, this study also found a correlation between LIPI subsets and clinical outcomes, with inferior outcomes in case of unfavorable baseline LIPI. The distribution of patients in good, intermediate and poor LIPI subsets was: $41 \%(\mathrm{~N}=77), 33.5 \%(\mathrm{~N}=63)$ and $6.9 \%$ $(\mathrm{N}=13)$, respectively. Median OS of patients was significantly different between LIPI groups $(\mathrm{P}<0.0001)$, with 20.8 mo (95\% CI, 14.9-not reached) for good LIPI, 7.3 mo (95\% CI: 4.4-12.9) for intermediate LIPI and 3.4 mo (95\% CI: 1.9 6.5) for poor LIPI. Median PFS was 6.6 mo (95\% CI: $4.7-$ 8.7), $5.1 \mathrm{mo}$ (95\% CI, 3.2-8.5) and $2.8 \mathrm{mo}$ (95\% CI: 1.8-3.9), respectively $(\mathrm{P}=0.07)$. DCR for good, intermediate and poor LIPI was $66 \%, 46 \%$ and $23 \%$, respectively $(\mathrm{P}=0.004)$. Poor LIPI was associated with shorter OS both in univariate (HR 3.12, 95\% CI: $2.12-4.60 ; \mathrm{P}<0.0001$ ) and multivariate (HR 3.67, 95\% CI: 1.96-6.86; $\mathrm{P}<0.0001)$ analyses. However, no independent association has been shown in case of PFS, probably because of a small number of patients in the poor LIPI subgroup (N=13). This paper reinforces the prognostic role of LIPI in NSCLC patients, however, in the absence of a comparator arm receiving chemotherapy, the potential predictive activity of LIPI cannot be evaluated.

Moreover, LIPI has been evaluated in patients with various tumor types treated with ICI, such as melanoma, head and neck, bladder, triple negative breast cancer, renal cell carcinoma, solid tumors harboring microsatellite instability and in patients with all tumor types included within phase I-II clinical trials $(8,15-17)$. These studies strongly suggest that the prognostic value of LIPI is tumor agnostic. Patients with poor LIPI have a poor benefit from ICI and thus, the evaluation of LIPI might identify subsets of patients with no or reduced benefit on ICI.

The available clinical results to date strongly suggests the prognostic role of the LIPI in advanced NSCLC patients, irrespective of the planned treatment. Further prospective studies should incorporate LIPI for validation as a prognostic marker and to clarify if there is any additional predictive role for ICI. Due to its prognostic value, we believe that LIPI could be used as a stratification factor in future clinical trials. Finally, although the potential predictive value of LIPI is not yet clarified, the identification of high-risk patients could still guide treatment selection by favoring the use of the most effective or combined therapy in a frontline setting.

\section{Acknowledgments}

Funding: None. 


\section{Footnote}

Conflicts of Interest: All authors have completed the ICMJE uniform disclosure form (available at http://dx.doi. org/10.21037/tlcr.2020.04.14). Dr. Mezquita reports personal fees from Roche Diagnostics, personal fees from Bristol-Myers Squibb, personal fees from Tecnofarma, personal fees from Roche, personal fees from AstraZeneca, other from Chugai, outside the submitted work. The other authors have no conflicts of interest to declare.

Ethical Statement: The authors are accountable for all aspects of the work in ensuring that questions related to the accuracy or integrity of any part of the work are appropriately investigated and resolved.

Open Access Statement: This is an Open Access article distributed in accordance with the Creative Commons Attribution-NonCommercial-NoDerivs 4.0 International License (CC BY-NC-ND 4.0), which permits the noncommercial replication and distribution of the article with the strict proviso that no changes or edits are made and the original work is properly cited (including links to both the formal publication through the relevant DOI and the license). See: https://creativecommons.org/licenses/by-nc-nd/4.0/.

\section{References}

1. Doroshow DB, Sanmamed MF, Hastings K, et al. Immunotherapy in Non-Small Cell Lung Cancer: Facts and Hopes. Clin Cancer Res 2019;25:4592-602.

2. Gauci ML, Lanoy E, Champiat S, et al. Long-Term Survival in Patients Responding to Anti-PD-1/PDL1 Therapy and Disease Outcome upon Treatment Discontinuation. Clin Cancer Res 2019;25:946-56.

3. Ferrara R, Mezquita L, Texier M, et al. Hyperprogressive Disease in Patients With Advanced Non-Small Cell Lung Cancer Treated With PD-1/PD-L1 Inhibitors or With Single-Agent Chemotherapy. JAMA Oncol 2018;4:1543-52.

4. Gandara DR, Reck M, Morris S, et al. Fast progression in patients treated with a checkpoint inhibitor (CPI) vs chemotherapy in OAK, a phase III trial of atezolizumab (ATEZO) vs docetaxel (DOC) in 2L+ NSCLC. Ann Oncol 2018;29:x39-43.

5. Charrier M, Mezquita L, Lueza B, et al. Circulating innate immune markers and outcomes in treatmentnaive advanced non-small cell lung cancer patients. Eur J
Cancer 2019;108:88-96.

6. Tumeh PC, Harview CL, Yearley JH, et al. PD-1 blockade induces responses by inhibiting adaptive immune resistance. Nature 2014;515:568-71.

7. Higgs BW, Morehouse CA, Streicher K, et al. Interferon Gamma Messenger RNA Signature in Tumor Biopsies Predicts Outcomes in Patients with Non-Small Cell Lung Carcinoma or Urothelial Cancer Treated with Durvalumab. Clin Cancer Res 2018;24:3857-66.

8. Benitez JC, Recondo G, Rassy E, et al. The LIPI score and inflammatory biomarkers for selection of patients with solid tumors treated with checkpoint inhibitors. Q J Nucl Med Mol Imaging 2020;64:162-74.

9. Mezquita L, Auclin E, Ferrara R, et al. Association of the Lung Immune Prognostic Index With Immune Checkpoint Inhibitor Outcomes in Patients With Advanced Non-Small Cell Lung Cancer. JAMA Oncol 2018;4:351-7.

10. Sorich MJ, Rowland A, Karapetis CS, et al. Evaluation of the Lung Immune Prognostic Index for Prediction of Survival and Response in Patients Treated With Atezolizumab for NSCLC: Pooled Analysis of Clinical Trials. J Thorac Oncol 2019;14:1440-6.

11. Kazandjian D, Gong Y, Keegan P, et al. Prognostic Value of the Lung Immune Prognostic Index for Patients Treated for Metastatic Non-Small Cell Lung Cancer. JAMA Oncol 2019;5:1481-5.

12. Ruiz-Bañobre J, Areses-Manrique MC, MosqueraMartínez J, et al. Evaluation of the lung immune prognostic index in advanced non-small cell lung cancer patients under nivolumab monotherapy. Transl Lung Cancer Res 2019;8:1078-85.

13. Riedl JM, Barth DA, Foris V, et al. External validation and longitudinal extension of the LIPI (Lung Immune Prognostic Index) for immunotherapy outcomes in advanced non-small cell lung cancer. Ann Oncol 2019;30:v514.

14. Mezquita L, Auclin E, Besse B. Letter to the Editor about Sorich et al. J Thorac Oncol 2019;14:e209.

15. Meyers DE, Stukalin I, Vallerand IA, et al. The Lung Immune Prognostic Index Discriminates Survival Outcomes in Patients with Solid Tumors Treated with Immune Checkpoint Inhibitors. Cancers (Basel) 2019. doi: 10.3390/cancers11111713.

16. Pauline P, Auclin E, Mezquita L, et al. Association of the Lung Immune Prognostic Index with outcome in patients with metastatic urothelial cancer treated with immune checkpoint inhibitor. J Clin Oncol 
2020;38:545.

17. Vuagnat P, Auclin E, Mezquita L, et al. Applicability of the LIPI score to metastatic microsatellite instability

Cite this article as: Aldea M, Benitez JC, Mezquita L. The Lung Immune Prognostic Index (LIPI) stratifies prognostic groups in advanced non-small cell lung cancer (NSCLC) patients. Transl Lung Cancer Res 2020;9(4):967-970. doi: 10.21037/tlcr.2020.04.14 high cancer patients treated with immune checkpoint inhibitors. Ann Oncol 2019;30:xi20. 